\title{
AUTOMATIC MEASURING SYSTEM FOR RAILROAD WHEELS
}

\author{
J. Figueiredo \\ CEM-IDMEC, Universidade Évora, R. Romão Ramalho, 59; 7000-671 Évora, \\ Portugal, jfig@uevora.pt
}

\begin{abstract}
This paper presents a vision system to measure the wear of the railroad wheels, developed for the Portuguese railway company, CP - Comboios Portugal.

The developed system is based on image processing techniques to measure automatically the railroad wheels. The wheel's profile is acquired by the illumination of the wheel through a secant horizontal light plane. The profile's image is processed by identifying the line of the greatest light gradient. The gradient's profile is then converted to the wheel's radial profile by means of a coordinate transformation model, which transforms the image plane into the wheel's radial plane
\end{abstract}

A 1:1 scale static prototype is built and the calibration and testing procedures are shown. Experimental results are presented and discussed. Future improvements on the developed prototype will allow dynamic measurements at slow speeds $(5 \mathrm{~km} / \mathrm{h})$, as the present developed image system was designed to eliminate distortions due to the wheel's movement.

Keywords: Artificial Vision, Automatic Measurement, Pattern recognition, Industrial applications

\section{Introduction}

The measurement of the railroad wheel's profile is a basic procedure in the regular maintenance of trains.

Railroad wheels have to be inspected on a regular time basis in order to evaluate the wear of the rolling surface. Wheels that have an excessive wear face a very high risk of derailing, causing a severe train accident.

The topic of wear of railroad wheels has been intensively studied during the last years and a large number of papers have been published (Gullers et al. 2011), (Froehling et al. 2008), (Kabo et al. 2011). Different occurrences of surface defects depend on a combination of factors, such as excessive vertical wheel-rail contact forces, increased vehicle speeds, rail surface conditions, rail joints and crossings, have been studied (Gullers et al. 2011), (Froehling et al. 2008). 